\title{
BRAF, KRAS and PIK3CA mutations in colorectal serrated polyps and cancer: Primary or secondary genetic events in colorectal carcinogenesis?
}

\author{
Sérgia Velho ${ }^{1}$, Cátia Moutinho ${ }^{1}$, Luís Cirnes ${ }^{1}$, Cristina Albuquerque ${ }^{2}$, \\ Richard Hamelin ${ }^{3}$, Fernando Schmitt ${ }^{1,4}$, Fátima Carneiro ${ }^{1,4,5}$, \\ Carla Oliveira ${ }^{1,4}$ and Raquel Seruca*1,4
}

\begin{abstract}
Address: ${ }^{1}$ Institute of Molecular Pathology and Immunology, University of Porto, Portugal, ${ }^{2}$ Centro de Investigação de Patobiologia MolecularCIPM, Instituto Português de Oncologia Francisco Gentil, Lisboa, Portugal, ${ }^{3}$ Inserm, UMRS 762, 75010, Paris, France, ${ }^{4}$ Faculty of Medicine, University of Porto, Portugal and ${ }^{5}$ Hospital of S. João, Porto, Portugal

Email: Sérgia Velho - svelho@ipatimup.pt; Cátia Moutinho - cmoutinho@ipatimup.pt; Luís Cirnes - lcirnes@ipatimup.pt; Cristina Albuquerque - mc.albuquerque@sapo.pt; Richard Hamelin - richard.hamelin@cephb.fr; Fernando Schmitt - fschmitt@ipatimup.pt; Fátima Carneiro - fcarneiro@ipatimup.pt; Carla Oliveira - carlaol@ipatimup.pt; Raquel Seruca* - rseruca@ipatimup.pt

* Corresponding author
\end{abstract}

Published: 9 September 2008

BMC Cancer 2008, 8:255 doi:10.1 186/147/-2407-8-255

This article is available from: http://www.biomedcentral.com//47/-2407/8/255

(c) 2008 Velho et al; licensee BioMed Central Ltd.

This is an Open Access article distributed under the terms of the Creative Commons Attribution License (http://creativecommons.org/licenses/by/2.0), which permits unrestricted use, distribution, and reproduction in any medium, provided the original work is properly cited.
Received: 20 February 2008

Accepted: 9 September 2008

\begin{abstract}
Background: BRAF, KRAS and PIK3CA mutations are frequently found in sporadic colorectal cancer (CRC). In contrast to KRAS and PIK3CA mutations, BRAF mutations are associated with tumours harbouring CpG Island methylation phenotype (CIMP), MLHI methylation and microsatellite instability (MSI). We aimed at determine the frequency of KRAS, $B R A F$ and PIK3CA mutations in the process of colorectal tumourigenesis using a series of colorectal polyps and carcinomas. In the series of polyps CIMP, MLHI methylation and MSI were also studied.
\end{abstract}

Methods: Mutation analyses were performed by PCR/sequencing. Bisulfite treated DNA was used to study CIMP and MLHI methylation. MSI was detected by pentaplex PCR and Genescan analysis of quasimonomorphic mononucleotide repeats. Chi Square test and Fisher's Exact test were used to perform association studies.

Results: KRAS, PIK3CA or BRAF occur in $71 \%$ of polyps and were mutually exclusive. KRAS mutations occur in $35 \%$ of polyps. PIK3CA was found in one of the polyps. V600E BRAF mutations occur in $29 \%$ of cases, all of them classified as serrated adenoma. CIMP phenotype occurred in $25 \%$ of the polyps and all were mutated for BRAF. MLHI methylation was not detected and all the polyps were microsatellite stable. The comparison between the frequency of oncogenic mutations in polyps and CRC (MSI and MSS) lead us to demonstrate that KRAS and PIK3CA are likely to precede both types of CRC. BRAF mutations are likely to precede MSI carcinomas since the frequency found in serrated polyps is similar to what is found in MSI CRC ( $P=0.9$ II2), but statistically different from what is found in microsatellite stable (MSS) tumours $(P=0.0191)$.

Conclusion: Our results show that BRAF, KRAS and PIK3CA mutations occur prior to malignant transformation demonstrating that these oncogenic alterations are primary genetic events in colorectal carcinogenesis. Further, we show that BRAF mutations occur in association with CIMP phenotype in colorectal serrated polyps and verified that colorectal serrated polyps and MSI CRC show a similar frequency of BRAF mutations. These results support that BRAF mutations harbour a mild oncogenic effect in comparison to KRAS and suggest that BRAF mutant colorectal cells need to accumulate extra epigenetic alterations in order to acquire full transformation and evolve to MSI CRC. 


\section{Background}

In microsatellite unstable (MSI) colorectal tumours, both sporadic and inherited forms, hundreds of thousands of mutations accumulate within repetitive sequences throughout the genome [1]. Although genes with repetitive sequences are clear targets in tumours with a defective mismatch repair system, mutations in non-repetitive sequences are also found in MSI tumours. An example of this is the occurrence of activating mutations of KRAS, PIK3CA and BRAF genes.

Activating mutations of $B R A F$ and $K R A S$ are frequently found in sporadic colorectal (CRC) cancer. BRAF mutations occur in about 10 to $18 \%$ of CRC overall and in 30 to $45 \%$ of MSI CRC $[2,3]$, more frequently in tumours harbouring MLH1 promoter hypermethylation [3] and with a CpG island methylation phenotype (CIMP) [4]. In microsatellite stable (MSS) CRC BRAF mutations are rare and whenever present are associated with advanced carcinomas [5]. KRAS mutations occur in both MSI (in about $20 \%$ ) $[3,6,7]$ and MSS (in about 35\%) subsets of sporadic CRC $[5,6]$. Within the MSI subset of CRC KRAS mutations do not associate with the presence of MLH1 promoter hypermethylation [3] or with the presence of CIMP [8]. PIK3CA mutations were identified in various tumour models [9]. In colorectal tumours, PIK3CA mutations are present in $14 \%$ to $25 \%$ of the cases $[9,10]$ and no differences in frequency and type of PIK3CA mutations were found between MSI and MSS subsets [10]. Further, whenever PIK3CA mutations occur in CRC they can occur in concomitance with activating KRAS-BRAF mutations, both in MSI and MSS tumours [10]. Nothing is known on the relationship between PIK3CA mutations and CIMP or MLH1 methylation in colorectal carcinomas.

The aim of the present study was to determine the timing of occurrence of KRAS, BRAF and PIK3CA mutations in the process of colorectal tumourigenesis and study the association of these mutational oncogenic events with CIMP, MLH1 methylation and MSI phenotype. In order to achieve our goal we evaluated the frequency and type of these epi/genetic events in a series of 17 colorectal polyps. Furthermore, we compared the frequency of KRAS, BRAF and PIK3CA mutations found in polyps with the frequency found in a series of 103 sporadic colorectal tumours, 50 MSI CRC and 53 MSS CRC, in order to understand the importance of these oncogenic events for the progression of the various types of colorectal cancer.

In conclusion, our results show that mutations of $B R A F$, KRAS and PIK3CA occur in non-malignant lesions of colorectum demonstrating that these oncogenic alterations are primary genetic events in colorectal carcinogenesis. Further, we show that BRAF mutations occur in association with CIMP phenotype in colorectal serrated polyps while KRAS mutations are found alone. These results support that $B R A F$ mutations harbour a mild oncogenic effect in comparison to KRAS and suggest that BRAF mutant colorectal cells need to accumulate extra epigenetic alterations in order to acquire full transformation.

\section{Methods}

Representative blocks of 17 colorectal polyps and 103 colorectal carcinomas formalin-fixed, paraffin embedded were retrieved from the Department of Pathology of the Hospital S. João. This study was a retrospective analysis and is in compliance with Helsinki declaration http:// www.wma.net/e/policy/b3.htm. The 103 sporadic CRC were previously classified for microsatellite status, 53 were MSS and 50 were MSI colorectal carcinomas [10]. The histological classification of the seventeen paraffin embedded colorectal polyps is listed in table 1 . None of the patients included in this study had a positive family history. DNA from these 17 polyps was extracted using the Invisorb Spin Tissue Mini Kit (Invitek). BRAF (exon 15) and KRAS (exon 1) were amplified by PCR and the presence of mutations was detected by direct sequencing. To perform methylation studies, DNA extracted from paraffin embedded lesions was submitted to bisulfite treatment. CIMP was determined in 12 cases by methylation specific PCR (MSP) using the new panel of five markers described by Weisenberger [4]. The polyps were classified as CIMP ( $\geq 3$ methylated markers), and CIMP-negative ( $\leq$ 2 methylated markers). We were able to analyze $M L H 1$ methylation in 7 samples. For that the promoter region of the gene was amplified using a set of CpG island flanking primers (sense 5'tttgtttttattggttggatattt, antisense 5 'ccttcaaccaatcacctcaatacct) followed by direct sequencing. Microsatellite instability studies were performed by genescan analyses of a PCR amplified panel of five mononucleotide markers (NR27, NR24, NR21, Bat25 and Bat26) using the Multiplex PCR Kit (Qiagen). MSI was considered when two or more microsatellite markers were altered. Association studies between the frequency of KRAS, PIK3CA and BRAF in colorectal polyps and MSI and MSS CRC were performed using the Chi Square test and Fisher's Exact test. A $P$-value $\leq 0.05$ was considered to be statistically significant.

\section{Results \\ KRAS, PIK3CA, and BRAF mutations occur prior to malignant transformation}

In our series of colorectal polyps, we found that mutations in KRAS, PIK3CA or BRAF are mutually exclusive and occur in the majority of these pre-malignant colorectal lesions. Mutations in KRAS, PIK3CA or BRAF occur in 12 (70.6\%) of the 17 colorectal polyps (Table 1 ).

KRAS mutations were found in 35.3\% (6/17) of polyps. KRAS mutant polyps showed three different types of 
Table I: Summary of the molecular and histological features of the colorectal polyps analyzed

\begin{tabular}{|c|c|c|c|c|c|c|c|c|}
\hline \multirow[b]{2}{*}{ Sample } & \multicolumn{6}{|c|}{ Molecular Classification } & \multicolumn{2}{|c|}{ Histological classification } \\
\hline & KRAS & BRAF & РІКЗСА & CIMP & MLH I methylation & Microsatellite instability & Polyp type & Dysplasia \\
\hline I & GI2D & - & - & Negative & UnMet & MSS & MHA & Mild \\
\hline 2 & GI2D & - & - & Negative & nd & MSS & $\mathrm{MHA}$ & Mild \\
\hline 3 & GI2V & - & - & nd & UnMet & MSS & $\mathrm{MHA}$ & Mild \\
\hline 4 & GI2V & - & - & Negative & nd & MSS & $\mathrm{HP}$ & - \\
\hline 5 & GI3D & - & - & Negative & nd & MSS & $\mathrm{HP}$ & - \\
\hline 6 & GI3D & - & - & Negative & nd & MSS & MHA & Mild \\
\hline 7 & - & V600E & - & Positive & UnMet & MSS & SA & Mild \\
\hline 8 & - & V600E & - & Positive & UnMet & MSS & SA & Mild \\
\hline 9 & - & V600E & - & Positive & nd & MSS & SA & Mild \\
\hline 10 & - & V600E & - & Negative & nd & MSS & SA & Mild \\
\hline 11 & - & V600E & - & nd & nd & MSS & SA & Moderate \\
\hline 12 & - & - & RI023Q & nd & nd & MSS & MHA & Mild \\
\hline 13 & - & - & - & Negative & UnMet & MSS & $\mathrm{MHA}$ & Mild \\
\hline 14 & - & - & - & Negative & UnMet & MSS & $\mathrm{HP}$ & - \\
\hline 15 & - & - & - & Negative & UnMet & MSS & MHA & Mild \\
\hline 16 & - & - & - & nd & nd & MSS & MHA & Mild \\
\hline 17 & - & - & - & nd & nd & MSS & HP & - \\
\hline
\end{tabular}

UnMet - unmethylated; nd - not determined; MHA - Mixed hyperplastic and adenomatous; HP - Hyperplastic polyp; SA - Serrated adenoma

mutations with the same frequency within the two most common hotspot codons (12 and 13): G12D (33.3\% - 2/ 6), G12V (33.3\% - 2/6) and G13D (33.3\% - 2/6). KRAS mutations were only found in polyps with some areas with dysplasia.

In contrast with the high frequency of KRAS mutations found in our series, PIK3CA mutations were observed in only one of the polyps analyzed (5.9\%) (Table 1). The PIK3CA mutation found was localized to exon 20 and affects amino acid 1023 leading to an arginine-glutamine substitution (R1023Q). This PIK3CA mutation resides in a hotspot exon for mutations that has been previously reported in a MSI colorectal tumour [10].

BRAF mutations were found in $29.4 \%(5 / 17)$ of the cases (Table 1). All BRAF mutations found in our series of polyps corresponded to the hotspot BRAF V600E and all polyps with BRAF mutations exhibited the serrated architecture.

\section{BRAF mutations occur in association with CIMP phenotype in colorectal polyps}

We studied the presence of CIMP phenotype, MLH1 promoter methylation and MSI phenotype and their association with the presence of KRAS, BRAF and PIK3CA mutations in the series of polyps.

Using the Weisenberger [4] panel of CIMP markers three of 12 polyps analyzed (25\%) were classified as CIMP ( $\geq 3$ methylated markers). All of the CIMP polyps were mutated for BRAF (Table 1). None of the CIMP polyps harbour KRAS mutations.

The methylation analysis of $M L H 1$ was possible in 7 of the 17 polyps. Two of these 7 polyps were mutated for KRAS, 2 harboured $B R A F$ mutations and 3 were wild-type for both genes. None of the 7 polyps studied harboured methylation in the promoter region of MLH1 (Table 1). Furthermore, we determined the MSI status of all polyps using the pentaplex set of microsatellite markers [11]. None of them were MSI.

\section{Colorectal serrated polyps and MSI CRC show a similar frequency of BRAF mutations}

In order to understand the importance of KRAS, PIK3CA and $B R A F$ mutations for the progression of the various types of colorectal cancer, we compared the frequency of these oncogenic events in the series of polyps and in 50 MSI and 53 MSS CRC (Figure 1).

Ten of the $50(20 \%)$ MSI and 21 of the 53 (39.6\%) MSS colorectal carcinomas exhibited KRAS mutations. The frequency of KRAS mutations found in the MSI and MSS CRC was not statistical different from the frequency found in colorectal polyps $(P=0.2014$ and $P=0.7497$, respectively).

The same was observed for PIK3CA mutations. MSI and MSS colorectal carcinomas harbour PIK3CA in 8 of the 50 $(16 \%)$ and in 6 of the $53(11.3 \%)$ cases, respectively. The frequency of mutations found in polyps, although lower 

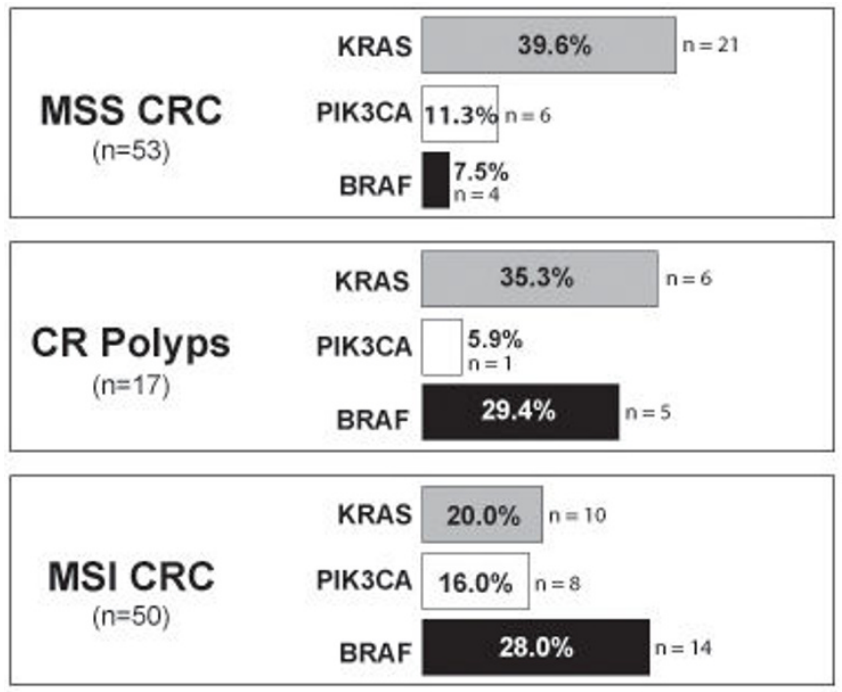

\section{Figure I}

KRAS, PIK3CA and BRAF mutation frequencies between MSI/MSS CRC and CR polyps. The frequency of KRAS, PIK3CA and BRAF mutations in MSI and MSS CRC was based on previous results of our group in Velho [10]. In brackets is represented the total of cases that were analyzed for each gene.

in comparison to colorectal carcinomas, is not statistically different from MSI $(P=0.4296$ - Fisher's Exact P-Value $)$ and MSS $(P>0.9999$ - Fisher's Exact P-Value) colorectal carcinomas.

Concerning the frequency of $B R A F$ alterations in colorectal carcinomas, 14 of the $50(28 \%)$ MSI and 4 of the 53 (7.5\%) in MSS colorectal tumours show BRAF mutations. We found that MSI CRC and colorectal serrated polyps show a similar frequency of $B R A F$ mutations $(P=0.9112)$. In contrast, the frequency of BRAF mutations in MSS carcinomas and colorectal polyps is significantly different $(P$ $=0.0191)$.

\section{Discussion}

Distinct types of colorectal polyps represent pre-malignant lesions of the intestinal tract. In our series of colorectal polyps we found the majority $(71 \%)$ harbour mutations in KRAS $(35.3 \%)$, PIK3CA $(5.9 \%)$ or BRAF $(29.4 \%)$ genes. Regarding KRAS mutations we show that these mutations occur in cases of hyperplasic polyps and in hyperplastic polyps with some areas of dysplasia in accordance to the recent reports of Wynter [12] and O'Brien [13] showing that KRAS mutations occur in premalignant lesions of the colorectum other than pure adenomas. When comparing the frequency of KRAS mutations found in our series of colorectal polyps with those observed within the MSS and MSI subsets of CRC, the frequency of KRAS mutations found in polyps is not statisti- cally different from the frequency observed in MSI and MSS colorectal tumours (Figure 1). Both observations highlight the role of KRAS activation in the initiation of the different types of sporadic CRC [12-14] besides its role in the adenoma-carcinoma multistep pathway of colorectal carcinogenesis [15].

PIK3CA mutations were found in only one of the polyps $(6 \%)$ but this frequency is not significantly different from what is observed in CRC independently of MSI status. The mutation found in our series of colorectal polyps was previously described in a colorectal tumour [10] suggesting that it harbours a pathogenic effect. Further, it is interesting to note that the PIK3CA alteration found in the single affected polyp is wild-type KRAS and BRAF case. This result suggests that mutant PIK3CA is likely to be an alternative rare genetic event to KRAS and BRAF in pre-malignant lesions of the colon.

The frequency of BRAF mutations in polyps $(30 \%)$ is higher than the frequency observed in overall CRC (17\%). This observation lead us to speculate that a fraction of BRAF mutant polyps do not progress to carcinoma and remain as non-malignant colorectal lesions as an endpoint stage. In accordance with this, it was demonstrated in mice that expression of active BRAF in the lungs lead to an initial burst of cell proliferation followed by growth arrest rarely inducing spontaneous progression to adenocarcinoma unless mice were deliberately engineered to lack the TP53 or Ink4a/Arf tumour suppressor genes [16]. This study and our data suggest that BRAF may need to cooperate with other factors (genetic or epigenetic) to progress to colorectal carcinoma, as it was previously proposed for human naevi $[17,18]$.

Noteworthy, the frequency of BRAF mutations in colorectal polyps is similar to the frequency observed in MSI CRC $(28 \%)(P=0.9112)$. These data raises the hypothesis that mutant BRAF triggers a distinct pathway of colorectal carcinogenesis leading to the formation of MSI colorectal tumours different from the adenoma-carcinoma sequence. Interestingly BRAF mutations occurred only in polyps with the serrated architecture supporting that $B R A F$ activation is pivotal in the serrated pathway of CRC as advanced previously by Jass $[19,20]$ and other authors $[13,14,21,22]$.

Analyzing the frequency of CIMP in colorectal polyps we have demonstrated that the results obtained are similar to what was described in CRC $[13,14,20,22]$ showing that BRAF mutations are associated with CIMP phenotype in the initial steps of carcinogenesis before malignant transformation. In contrast, KRAS mutations were only associated to with low levels of methylation as it was previously observed in primary colorectal tumours $[19,23]$, demon- 
strating that KRAS mutations are associated to mild effects in the methylation profile during colorectal carcinogenesis. These results support that BRAF mutations harbour a mild oncogenic effect in comparison to KRAS and suggest $B R A F$ mutant colorectal cells need to accumulate extra epigenetic alterations in order to acquire full transformation.

MSI and loss of expression of MLH1 due to promoter hypermethylation are frequently associated with $B R A F$ mutation and CIMP in MSI sporadic colorectal tumours [2-4]. In our series of colorectal polyps we did not detected MLH1 promoter hypermethylation which is in agreement with the fact that none of the analyzed polyps harboured MSI. Our data is in agreement with the results previously described in serrated adenomas described by Sawyer [24] demonstrating that BRAF and CIMP precedes MSI as proposed by the model advanced by O'Brien [14]. The late acquisition of MLH1 methylation leading to the accumulation of mutations throughout the genome targeting important genes (tumour suppressor genes or proto-oncogenes) involved in the control of cellular homeostasis may bridge the transition from a pre-malignant to a malignant lesion.

\section{Conclusion}

In conclusion, our results show that mutations of KRAS and PIK3CA occur in non-malignant lesions of colorectum and the frequency of these mutations is not distinct in polyps and MSS and MSI CRC demonstrating that these oncogenic alterations are primary genetic events in the two subsets of colorectal carcinogenesis. Further, we show that BRAF mutations occur in association with CIMP phenotype in colorectal serrated polyps while KRAS mutations are found alone. Further we show that only MSI CRC and colorectal serrated polyps harbour similar frequency of BRAF mutations. These results support that BRAF mutations harbour a mild oncogenic effect in comparison to KRAS and suggest that BRAF mutant colorectal cells need to accumulate extra epigenetic alterations in order to acquire full transformation and evolve to MSI CRC.

\section{Competing interests}

The authors declare that they have no competing interests.

\section{Authors' contributions}

SV did BRAF and PIK3CA mutation screening, performed methylation studies (CIMP and MLH1 methylation) and drafted the manuscript. CM did KRAS mutation screening. LC performed MSI analyses. CA collected patient material with distinct subtypes of colorectal polyps. RH analyzed the MSI status of CRC. FS and FC were responsible for the histological classification of CRC. CO did BRAF and KRAS screening in CRC and helped to draft the manuscript. RS conceived and designed the study, analyzed the results and helped to draft the manuscript. All authors read and approved the final manuscript.

\section{Acknowledgements}

To the FCT grant POCTI/SAU-OBS/5692I/2004 and PTDC/SAU-OBD/ $68310 / 2006$

\section{References}

I. Ionov Y, Peinado M, Malkhosyan S, Shibata D, Perucho M: Ubiquitous somatic mutations in simple repeated sequences reveal a new mechanism for colonic carcinogenesis. Nature 1993 , 363(6429):558-56I.

2. Lubomierski N, Plotz G, Wormek M, Engels K, Kriener S, Trojan J, Jungling B, Zeuzem S, Raedle J: BRAF mutations in colorectal carcinoma suggest two entities of microsatellite-unstable tumors. Cancer 2005, I04(5):952-961.

3. Domingo E, Espín E, Armengol M, Oliveira C, Pinto M, Duval A, Brennetot C, Seruca R, Hamelin R, Yamamoto H, Schwartz SJ: Activated BRAF targets proximal colon tumors with mismatch repair deficiency and MLHI inactivation. Genes Chromosomes Cancer 2004, 39(2): 138-142.

4. Weisenberger D, Siegmund K, Campan M, Young J, Long T, Faasse $M$, Kang G, Widschwendter M, Weener D, Buchanan D, Koh H, Simms L, Barker M, Leggett B, Levine J, Kim M, French A, Thibodeau S, Jass J, Haile R, Laird P: CpG island methylator phenotype underlies sporadic microsatellite instability and is tightly associated with BRAF mutation in colorectal cancer. Nat Genet 2006, 38(7):787-793.

5. Oliveira C, Velho S, Moutinho C, Ferreira A, Preto A, Domingo E, Capelinha A, Duval A, Hamelin R, Machado J, Schwartz SJ, Carneiro $F$, Seruca R: KRAS and BRAF oncogenic mutations in MSS colorectal carcinoma progression. Oncogene 2007, 26(I):158-163.

6. Oliveira C, Westra J, Arango D, Ollikainen M, Domingo E, Ferreira A, Velho S, Niessen R, Lagerstedt K, Alhopuro P, Laiho P, Veiga I, Teixeira M, Ligtenberg M, Kleibeuker J, Sijmons R, Plukker J, Imai K, Lage P, Hamelin R, Albuquerque C, Schwartz SJ, Lindblom A, Peltomaki P, Yamamoto H, Aaltonen L, Seruca R, Hofstra R: Distinct patterns of KRAS mutations in colorectal carcinomas according to germline mismatch repair defects and $\mathrm{hMLHI}$ methylation status. Hum Mol Genet 2004, I 3(19):2303-23II.

7. Oliveira C, Pinto M, Duval A, Brennetot C, Domingo E, Espin E, Armengol M, Yamamoto H, Hamelin R, Seruca R, Schwartz $S$ Jr: BRAF mutations characterize colon but not gastric cancer with mismatch repair deficiency. Oncogene 2003, 22(57):9192-9196.

8. Samowitz W, Albertsen H, Herrick J, Levin T, Sweeney C, Murtaugh $M$, Wolff R, Slattery M: Evaluation of a large, population-based sample supports a CpG island methylator phenotype in colon cancer. Gastroenterology 2005, I 29(3):837-845.

9. Samuels $Y$, Wang Z, Bardelli A, Silliman N, Ptak J, Szabo S, Yan H, Gazdar A, Powell S, Riggins G, Willson J, Markowitz S, Kinzler K, Vogelstein B, Velculescu V: High frequency of mutations of the PIK3CA gene in human cancers. Science 2004, 304(5670):554.

10. Velho S, Oliveira C, Ferreira A, Ferreira A, Suriano G, Schwartz SJ, Duval A, Carneiro F, Machado J, Hamelin R, Seruca R: The prevalence of PIK3CA mutations in gastric and colon cancer. Eur J Cancer 2005, 4 I (I I): 1649-1654.

II. Suraweera N, Duval A, Reperant M, Vaury C, Furlan D, Leroy K, Seruca R, lacopetta B, Hamelin R: Evaluation of tumor microsatellite instability using five quasimonomorphic mononucleotide repeats and pentaplex PCR. Gastroenterology 2002, I 23(6): | 804-|8II.

12. Wynter C, Walsh M, Higuchi T, Leggett B, Young J, Jass J: Methylation patterns define two types of hyperplastic polyp associated with colorectal cancer. Gut 2004, 53(4):573-580.

13. O'Brien M: Hyperplastic and serrated polyps of the colorectum. Gastroenterol Clin North Am 2007, 36(4):947-968.

14. O'Brien M, Yang S, Mack C, Xu H, Huang CS, Mulcahy E, Amorosino $M$, Farraye $F$ : Comparison of microsatellite instability, CpG island methylation phenotype, BRAF and KRAS status in serrated polyps and traditional adenomas indicates separate pathways to distinct colorectal carcinoma end points. $\mathrm{Am} J$ Surg Pathol 2006, 30(12):149I-I50I. 
15. Vogelstein B, Fearon E, Hamilton S, Kern S, Preisinger A, Leppert M, Nakamura $Y$, White R, Smits A, Bos J: Genetic alterations during colorectal-tumor development. N Engl J Med 3 19(9):525-532. 1988, Sep I

16. Dankort D, Filenova E, Collado M, Serrano M, Jones K, McMahon M: A new mouse model to explore the initiation, progression, and therapy of BRAFV600E-induced lung tumors. Genes Dev 2007, 2 I (4):379-384.

17. Wajapeyee N, Serra R, Zhu X, Mahalingam M, Green M: Oncogenic BRAF Induces Senescence and Apoptosis through Pathways Mediated by the Secreted Protein IGFBP7. Cell 2008, 132:363-374.

18. Michaloglou C, Vredeveld L, Soengas M, Denoyelle C, Kuilman T, Horst Cvd, Majoor D, Shay J, Mooi W, Peeper D: BRAFE600-associated senescence-like cell cycle arrest of human naevi. Nature 2005, 436(705 I):720-724.

19. Jass J: Classification of colorectal cancer based on correlation of clinical, morphological and molecular features. Histopathology 2007, 50(1): I13-130.

20. Jass J: Colorectal cancer: a multipathway disease. Crit Rev Oncog 2006, I 2(3-4):273-287.

21. Minoo P, Moyer M, Jass J: Role of BRAF-V600E in the serrated pathway of colorectal tumourigenesis. J Pathol 2007, 2I 2(2): 124-133.

22. Kambara T, Simms L, Whitehall V, Spring K, Wynter C, Walsh M, Barker M, Arnold S, McGivern A, Matsubara N, Tanaka N, Higuchi T, Young J, Jass J, Leggett B: BRAF mutation is associated with DNA methylation in serrated polyps and cancers of the colorectum. Gut 2004, 53(8): | | 37- I | 44

23. Shen L, Toyota M, Kondo Y, Lin E, Zhang L, Guo Y, Hernandez N, Chen X, Ahmed S, Konishi K, Hamilton S, Issa J: Integrated genetic and epigenetic analysis identifies three different subclasses of colon cancer. Proc Natl Acad Sci USA 104(47): $18654-18659$. 2007, Nov 20;

24. Sawyer E, Cerar A, Hanby A, Gorman P, Arends M, Talbot I, Tomlinson I: Molecular characteristics of serrated adenomas of the colorectum. Gut 2002, 5 I(2):200-206.

\section{Pre-publication history}

The pre-publication history for this paper can be accessed here:

http://www.biomedcentral.com/1471-2407/8/255/pre

pub

Publish with Biomed Central and every scientist can read your work free of charge

"BioMed Central will be the most significant development for disseminating the results of biomedical research in our lifetime. "

Sir Paul Nurse, Cancer Research UK

Your research papers will be:

- available free of charge to the entire biomedical community

- peer reviewed and published immediately upon acceptance

- cited in PubMed and archived on PubMed Central

- yours - you keep the copyright
BioMedcentral 